Brazilian Journal

of Chemical

Engineering

\title{
PVC MEMBRANES PREPARED VIA NON-SOLVENT INDUCED PHASE SEPARATION PROCESS
}

\author{
Liana F. Padilha ${ }^{1 *}$ and Cristiano P. Borges ${ }^{1}$ \\ ${ }^{1}$ Universidade Federal do Rio de Janeiro, Centro de Tecnologia, Programa de Engenharia Química, Rio de Janeiro, RJ, Brasil. \\ E-mail: liana@peq.coppe.ufrj.br, ORCID: 0000-0001-8999-3616
}

(Submitted: March 9, 2017 ; Revised: January 9, 2018 ; Accepted: January 29, 2018)

\begin{abstract}
Polyvinylchloride (PVC) based membranes are prepared via a phase inversion method using N,Ndimethylacetamide (DMAc) as solvent and water as precipitation bath. Polyvinylpyrrolidone (PVP) and lithium nitrate $\left(\mathrm{LiNO}_{3}\right)$ are used as additives. Experimental cloud point data and solution viscosity measurements are evaluated. Precipitation rates, transport properties and membrane morphology are quantified. Membranes with different morphologies and transport properties are prepared by changing the additive and its concentration, changing the PVC concentration and by varying the exposure time to the environment before immersion. An augment in PVC concentration increases solution viscosity, reduces precipitation rate and water permeability $\left(\mathrm{J}_{\mathrm{w}}\right)$, but it does not affect the instantaneous precipitation mechanism. PVC solutions with additives present higher viscosity values, slower light transmittance decay and membranes with higher $\mathrm{J}_{\mathrm{w}}$ (highest: $\sim 1,350 \mathrm{~L} \cdot \mathrm{h}^{-1} \cdot \mathrm{m}^{-2}$. bar $\left.^{-1}\right)$. $\mathrm{LiNO}_{3}$ in the polymeric solution results in delayed demixing. A combination of high PVP concentration and environment exposure time changes the membrane morphology, suggesting spinodal demixing.

Keywords: PVC membranes; Phase inversion method; Membrane morphology; PVC transport properties.
\end{abstract}

\section{INTRODUCTION}

The non-solvent induced phase separation process (NIPS), better known as immersion precipitation technique, was first reported by Loeb and Sourirajan in the early 60 s to obtain high flux reverse osmosis membranes. Since then several studies were developed to improve this technique. In this process, a polymer solution is induced to separate into two liquid phases, one polymer rich and anotherpolymer poor, by changing its composition, temperature or by chemical reactions. The polymer concentration increases in the solution due to mass transfer of solvent to the precipitation bath. Nucleation of a polymer lean phase takes place in the polymer solution, accelerating the increase in the polymer concentration in the surrounding solution until its glass transition temperature is reached. Therefore, the membrane morphology will be defined by the competition between the liquid-liquid phase separation process and the viscous effects promoted by the increase of polymer concentration in the rich phase. Thus, the mass transfer rate between phases and non-solvent bath is the most effective way to control the membrane morphology (Baker, 2004; Mulder, 1991; Carvalho, 2005; Guillen, 2011).

Polymeric membranes can be used in several separation processes such as, microfiltration (MF), ultrafiltration (UF), nanofiltration (NF), and the advantages over traditional separation techniques are well known in the academic literature. Most of the membrane processes are pressure driven and can be distinguished by the size of particle that will be retained by the membrane. This characteristic is related with the pore-size of the membrane (membrane morphology), which is an intrinsic result of membrane synthesis (Baker, 2004; Guillen, 2011; Porter, 1990).

Membranes with a variety of pore-sizes can be produced by NIPS varying the polymer and its concentration, the type and concentration of additive, the precipitation medium and temperature. Most

\footnotetext{
* Corresponding author: Liana F. Padilha - E-mail: liana@peq.coppe.ufrj.br
} 
industrial membranes are produced by the NIPS technique, using water as non-solvent in a precipitation bath (Mei, 2011).

Several studies have been dedicated to understand the relation between membrane morphology and precipitation conditions of the polymer solution (Fen'ko et al., 2011; Frommer and Messalem, 1973; Machado et al., 1999; Mckelvey and Koros, 1996; Wijmans et al., 1985, Maghsoud et al., 2014).

However, the correlation between the morphology of the membranes and their transport properties is still a persistent question and few studies are intent to fill this gap. The direct influence of the membrane morphology on the transport properties is difficult to predict since the presence and size of pores in the membrane matrix is not a guarantee of high fluxes or better solute rejections.

A variety of soluble polymers can be employed to synthesize membranes by NIPS. Polyvinylchloride (PVC) is an outstanding material due to its well known stiffness, chemical and mechanical resistance and low cost. Furthermore, PVC has good affinity with several solvents used in the NIPS technique, such as N-methylpyrrolidone (NMP), N,N-dimethylformamide (DMF) and N,N-dimethylacetamide (DMAc) (Mei et al., 2011; . Bodzek and Konieczny, 1991; Okuno et al., 1993).

Hirose et al. (1979 and 1982) prepared flat sheet PVC membranes using DMF as solvent and methanol as precipitation bath. The morphology of the PVC membranes was explained through solution cloud point measurements.

Bodzek and Konieczny (1991) studied PVC membrane formation by NIPS using water as precipitation bath. The authors varied the molar mass and concentrations of PVC. They found that the molar mass affected the initial solution viscosity, but did not influence the binodal curve location. However, smaller PVC molar mass led to a faster precipitation and more porous membranes. On the other hand, higher PVC concentrations also accelerated the precipitation, but led to less porous membranes. The influences of PVC concentration on membrane morphology were also explained in literature (Mulder, 1991; Okuno et al., 1993; Babu and Gaikar, 1999; Guillen, 2011).

Okuno et al. (1993) synthesized PVC membranes using a mixture of tetrahydrofuran (THF) and alcohols as solvent, obtaining different solution viscosities. As the solution viscosity decreased more porous membranes were formed. These authors obtained similar results by reducing the PVC molar mass. Hence, it was concluded that the morphology of PVC membranes is more dependent on kinetic than on thermodynamic effects.

Kawai and Lee (1997) investigated the influence on membrane formation of different solvents in the casting solution. The phase diagrams of the systems were obtained by determining the equilibrium phase composition. Besides, the influence of moisture and temperature were also evaluated. They showed that reducing the outflow of THF, the polymer lean phase nuclei grow along the membrane cross section. Similar results were observed with PVC/DMF solution. When phase separation occurs by immersion in a water bath, a thin layer is formed instantaneously at the top surface of the solution, diminishing the outflow of DMF and allowing pore growth through the membrane cross section.

Babu and Gaikar (1999) studied the morphology and transport properties of PVC and CPVC membranes and their blends. They used DMF as solvent and varied the polymer concentration between 10 and $12 \mathrm{wt} \%$, precipitating the membranes in a water/alcohol bath. They found that an exposure time to the environment between 10-15 seconds before immersion in the precipitation bath changes the morphology and therefore the transport properties. The membranes exhibited water permeation flux in the range of 100 to $950 \mathrm{~L}$ $\mathrm{m}^{-2} \mathrm{~h}^{-1}$ with bovine serum albumin (BSA) rejections varying from 85 to $99 \%$. In another article Babu and Gaikar (2000) reported that polyvinylpyrrolidone (PVP - Molecular Weight 68,000 Da) as additive in the polymer solution, improved the hydrophilicity of PVC membranes. Higher PVP concentration in the polymer solution led to a reduction in BSA rejection, but to an increase in the permeate flux. The authors mentioned that the main limitation of PVC membranes lies in its hydrophobic character, which could be contoured by the use of additives with suitable properties to improve the membrane hydrophilicity.

To improve the membrane hydrophilicity Peng et al. (2006) investigated blends of polyvinylbutyral (PVB) and PVC and found that a PVC/PVB ratio of 9:1 improved both water flux and protein rejection $(\sim 99 \%)$, although an increase in PVB concentration resulted in lower water flux.

Mei et al. (2011) studied the influence of different additives (PVP, polyethylene glycol - PEG or sucrose) on the polymer solution viscosity, phase diagrams and membrane morphology. For all additives, they observed an increase in the solution viscosity, which could lead to flux reduction during mass transfer of solvent and non-solvent in the precipitation bath. Furthermore, a higher additive concentration led to phase separation in the polymer solution. The authors emphasized that kinetics and thermodynamics play an important role in membrane morphology and should be deeply analyzed in each case.

Maghsoud et al. (2014) prepared PVC membranes with a mixture of solvents (DMF and tetrahydrofuran - THF) to better understand the influence of thermodynamics and kinetics in membrane morphology and transport properties. After immersion in a water precipitation bath, the authors observed 
delayed and instantaneous demixing of $\mathrm{PVC} / \mathrm{THF} /$ water and $\mathrm{PVC} / \mathrm{DMF} /$ water systems, respectively. Addition of $\mathrm{THF}$ to $\mathrm{PVC} / \mathrm{DMF} /$ water solutions decreased the precipitation time, but the cloud point curve indicated that more water was admitted into the solution before precipitation. The authors presented a model to predict the cloud point composition and precipitation times as a function of $\mathrm{DMF} / \mathrm{THF}$ ratio in the solution. The model showed good agreement for cloud point and precipitation time, but was far from predicting membrane morphology and transport properties. According to the authors, changes in membrane performance and morphology cannot be explained just taking into account the thermodynamic properties of the polymer solution.

Machado et al. (1999) showed that the membrane formation mechanisms depend on the precipitation kinetics. Preparing polysulfone/PVP membrane, the authors reported an oscillatory behavior in macrovoid formation as a function of the precipitation rate. The authors concluded that phase separation kinetics should be taken into account to understand the membrane formation mechanism.

Reuvers (1987) presented a comparison between light transmittance and polymer concentration. The author showed that it is possible to control the membrane morphology by varying polymer concentration and several other process parameters and to estimate its behavior by light transmittance experiments. However, none of the new articles uses these experiments as a factor of verification.

Despite all studies, some questions remain, such as the correlation between the precipitation rate and membrane morphology, or transport properties, and a more thorough study has not yet been presented. In this study, PVC membranes were prepared by phase inversion through the immersion precipitation method, measuring precipitation rate, exposure time before immersion, miscibility region in the phase diagram and solution viscosity and relating with membrane morphology and transport properties. DMAc was used as solvent and water as non-solvent. PVP and $\mathrm{LiNO}_{3}$ were also evaluated as additives in the polymer solution. Membranes with different morphologies and transport properties could be prepared by choosing the additive and its concentration, changing PVC concentration and exposure time to the environment before immersion. An attempt to correlate the membrane synthesis conditions with the final transport properties was accomplished.

\section{EXPERIMENTAL}

\section{Materials}

Polyvinyl chloride (PVC) was obtained from Braskem S.A. with molar mass of $60 \mathrm{kDa}$, dried at $60{ }^{\circ} \mathrm{C}$ for at least 24 hours. Polyvinylpyrrolidone,
(PVP - K90), lithium nitrate $\left(\mathrm{LiNO}_{3}\right)$ and N,Ndimethylacetamide (DMAc, 99.95\% purity) were acquired from Sigma-Aldrich and used as additives and solvent, respectively, without further purification. Bovine serum albumin (BSA) with $66 \mathrm{kDa}$ of molar mass (Sigma-Aldrich) was used for the characterization of membrane rejection.

\section{Phase diagrams}

The cloud point was estimated by the rapid titration method described in Wijmans et al. (1985). PVC solutions with $11,14,17$ and $20 \mathrm{wt} \%$ were prepared using DMAc as solvent. The solution was placed into a sealed flask with magnetic stirrer at $30{ }^{\circ} \mathrm{C}$. Small amounts of distilled water were slowly added to the solution by using a $1 \mathrm{~mL}$ syringe, and the solution was stirred for at least 1 hour. The addition of water was continued until the solution visually became cloudy and, then, the final composition was calculated. The additive ( $\mathrm{PVP}$ or $\mathrm{LiNO}_{3}$ ) concentration was also varied keeping PVC concentration at $11 \mathrm{wt} \%$.

\section{Membrane Preparation}

The membranes were prepared by the NIPS method as described before (Mei et al., 2011). PVC at 11, 14, 17 and $20 \mathrm{wt} \%$ was dissolved in DMAc at $80^{\circ} \mathrm{C}$. The polymer solutions were cast on a glass plate using a stainless steel blade and immersed into a microfiltrated water precipitation bath at room temperature (ca. 25 ${ }^{\circ} \mathrm{C}$ ). The solution was instantaneously immersed into the precipitation bath or exposed to the lab environment for $30 \mathrm{~s}, 60 \mathrm{~s}$ or until complete precipitation by moisture (60\%r.h.) absorption (infinite time). The membranes were removed from the glass plate and kept overnight in a water bath to remove residual solvent. Hereafter, the membranes were kept at least 24 hours in a 40 $\mathrm{wt} \%$ glycerol bath to avoid porous collapse and dried at room temperature.

\section{Viscosity measurement}

The viscosity of the solutions was measured with a Brookfield Digital Viscometer LV series at various temperatures, polymers and additive compositions. The measurements were taken in the pseudoNewtonian range.

\section{Membrane morphology characterization}

Membrane morphologies were observed with a scanning electron microscope (FEI Co. Quanta 200), after covering the surface with sputtered gold (Jeol mod. 1500). The membrane samples were broken after cooling in liquid nitrogen to minimize deformation during fracture.

\section{Precipitation behavior}

Light transmittance measurements were carried out to obtain the onset of phase separation in the NIPS 
process, as well as the precipitation behavior based on transmittance reduction with time, as described in Fen'ko et al. (2011). The experiments were carried out using an infrared light beam collimated into the casting film. A sensor placed under the glass plate during the precipitation could continuously detect light variation. The detector signals were acquired by a computer and stored for further analysis.

\section{Transport Properties}

PVC membranes were evaluated according to their transport properties by UF experiments in a flat sheet module with an effective area of $18.1 \mathrm{~cm}^{2}$. Distilled and microfiltrated water was fed at constant pressure (1 bar) and room temperature. The permeability $\left(\mathrm{J}_{\mathrm{w}}, \mathrm{L}\right.$ $\mathrm{h}^{-1} \mathrm{~m}^{-2}$ bar $^{-1}$ ) was obtained as follows:

$$
\mathrm{J}_{\mathrm{w}}=\frac{\mathrm{Q}_{\mathrm{w}}}{\mathrm{A} \cdot \Delta \mathrm{P}}
$$

where $Q_{w}$ is the volumetric permeate flow rate $(\mathrm{L} / \mathrm{h})$; $\Delta \mathrm{P}$ is the pressure difference through the membrane (bar); A is the membrane surface area $\left(\mathrm{m}^{2}\right)$. The initial $\mathrm{J}_{\mathrm{BSA}}$ was employed when BSA solution was used as feed in UF experiments and calculated in the same manner exhibited in Equation 1.

The membrane separation property was evaluated by measuring the rejection of bovine serum albumin (BSA) with $66 \mathrm{kDa}$ of molar mass (Eq. 2). The permeation was performed with a solution containing $0.05 \mathrm{wt} \%$ of BSA.

$\mathrm{R}=1-\left(\frac{\mathrm{C}_{\mathrm{p}}}{\mathrm{C}_{\mathrm{f}}}\right)$

where $\mathrm{R}$ is the BSA rejection (\%), $\mathrm{C}_{\mathrm{p}}$ and $\mathrm{C}_{\mathrm{f}}$ are the BSA concentration in the permeate and feed solution, respectively. The solute concentration was measured by a UV-spectrophotometer (UV-Vis Analyst, UV1803) based on the absorbance of the solute at 278 nm wavelength.

\section{RESULTS AND DISCUSSION}

\section{Phase Diagram}

The behavior of the phase inversion process during immersion of the cast polymer solution in the precipitation bath can be related to cloud point measurements, since it is representative of the binodal curve in the phase diagram. This curve is dependent on thermodynamic properties of the three-component system and provides insight for the precipitation mechanisms. Figure 1 shows the phase diagram for the $\mathrm{PVC} / \mathrm{DMAc} /$ Water system, and Table 1 presents the

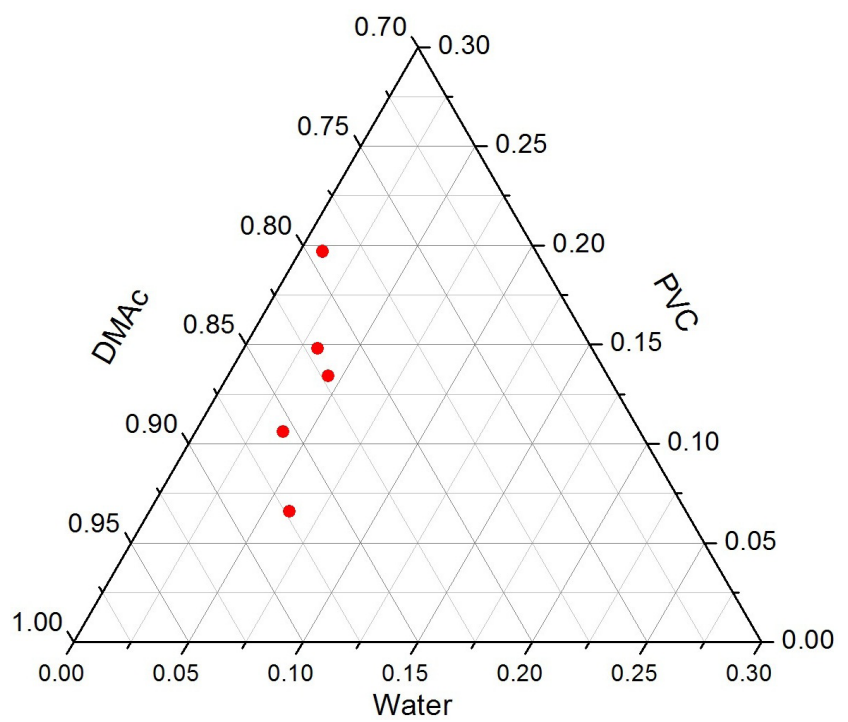

Figure 1. Cloud point data of PVC/DMAc/Water system.

Table 1. Cloud point composition for PVC in DMAc with $\mathrm{PVP}$ or $\mathrm{LiNO}_{3}$ as additive.

\begin{tabular}{ccccc}
\hline Additive & $\begin{array}{c}\text { PVC } \\
(\mathbf{w t} \%)\end{array}$ & $\begin{array}{c}\text { Additive } \\
(\mathbf{w t} \%)\end{array}$ & $\begin{array}{c}\text { DMAc } \\
(\mathbf{w t} \%)\end{array}$ & $\begin{array}{c}\text { Water } \\
\mathbf{( w t \% )}\end{array}$ \\
\hline- & 10.6 & - & 85.6 & 3.8 \\
& 10.6 & 4.9 & 80.3 & 4.3 \\
PVP & 10.7 & 7.0 & 79.5 & 2.8 \\
& 10.8 & 9.9 & 76.5 & 2.8 \\
& 10.8 & 3.0 & 83.9 & 2.3 \\
$\mathrm{LiNO}_{3}$ & 10.8 & 4.9 & 82.4 & 1.8 \\
& 10.9 & 6.9 & 80.2 & 2.0 \\
\hline
\end{tabular}

water content at the cloud point for the ternary system and with the addition of PVP or LiNO . It is observed that the miscibility region of the PVC solution is limited; even at low PVC concentrations the maximum water in the solution is slightly higher than $5 \mathrm{wt} \%$. According to Reuvers (1987), this behavior favors an instantaneous precipitation mechanism in the polymer solution top layers, and macrovoid formation in regions beneath.

Addition of $\mathrm{LiNO}_{3}$ to the PVC solution, as reported in Table 1, shifts the cloud point to the polymer-solvent axis, which was also observed with high concentrations of PVP. It is expected that the precipitation of the polymer solution becomes faster in this condition when compared with the solution without additive.

\section{Membrane formation: ternary system}

In the PVC/DMAc/Water system, the solvent and non-solvent exchange during solution precipitation plays an important role in the morphology and transport properties (permeability and rejection) of the membrane. The polymer concentration and water influx have strong influence on the membrane pore size, particularly in the instantaneous precipitation mechanism. Besides the thermodynamic properties of 
the system, the path and the velocity of the precipitation would also contribute to define the final membrane characteristics.

Figure 2 shows the cross section morphologies of membranes prepared from polymer solutions with 11 , 14,17 and $20 \mathrm{wt} \%$ of PVC and precipitated in a water bath.

Figure 3 presents the micrograph of the upper surface of the membranes prepared from solutions with 11 and $20 \mathrm{wt} \%$ of PVC. The densification of this region at higher $\mathrm{PVC}$ concentration is clearly
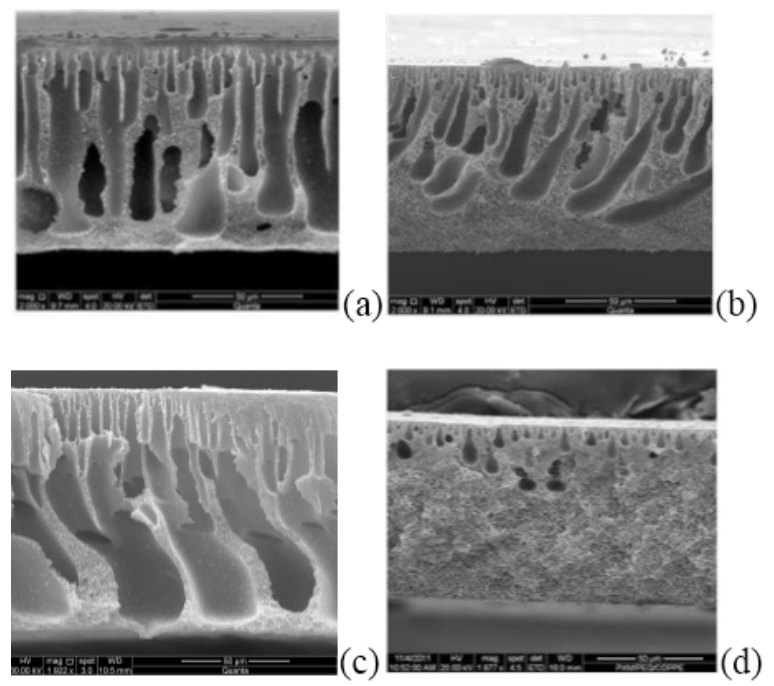

Figure 2. Cross section morphology of membranes synthesized from PVC/DMAc solution by instantaneous immersion in a water precipitation bath. PVC concentration: (a) $11 \mathrm{wt} \%$; (b) $14 \mathrm{wt} \%$; (c) 17 wt $\%$; (d) $20 w t \%$.
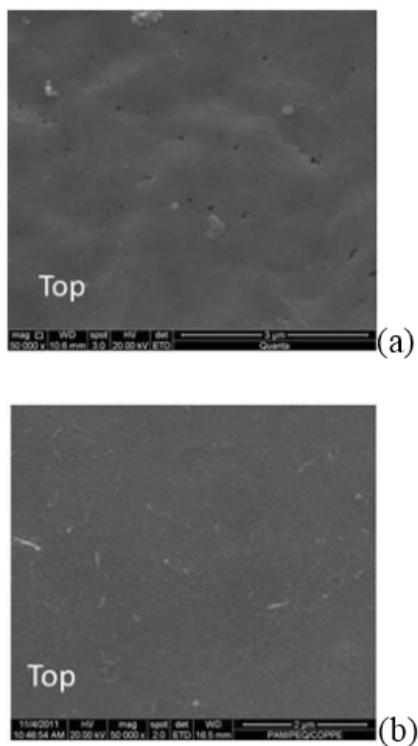

Figure 3. Upper surface morphology of the membranes synthesized from PVC/DMAc solution by instantaneous immersion in a water precipitation bath. PVC concentration: (a) $11 \mathrm{wt} \%$ and (b) $20 \mathrm{wt} \%$. observed. This densification is promoted when the solution approaches the region where the viscous effect predominates.

Mei and co-workers (2011) suggested that polymer solution viscosity is a key parameter in membrane formation, affecting the morphology and transport properties. In general, they showed that higher viscosity solutions prevent macrovoid formation and vice versa. Figure 4 shows viscosity measurements for the polymer solutions studied as a function of temperature. Hence, the sponge-like morphology predominant in membranes synthesized with $20 \mathrm{wt} \%$ of PVC (Figure 2) may be also a consequence of higher viscosity hindering nuclei expansion. However, one should consider that the solution viscosity along the solution depth changes as a function of the mass transfer with the non-solvent bath and the precipitation path.

Actually, it seems that in membrane formation by the phase inversion process the final morphology results from compound affinity, explained by thermodynamics, and competition between mass transfer and precipitation rate. Understanding the role and predominance of each mechanism should allow predicting the resultant membrane morphology.

An effort to elucidate the influence of mass transfer on membrane morphology was made in this study, measuring the precipitation rate by light transmittance experiments, as described above. Figure 5 shows the light transmission intensity decay of PVC/DMAc solutions as a function of the immersion time in a water precipitation bath. As can be seen, all curves decreased very rapidly, without any delay time, just after immersion in the precipitation bath, which points to an instantaneous precipitation mechanism. After one second (1s), the light transmittance intensity tends to decay smoothly, reaching a plateau at $20-50 \%$ of the initial light transmittance intensity. Figure 5(b) presents the light transmittance intensity decay for the

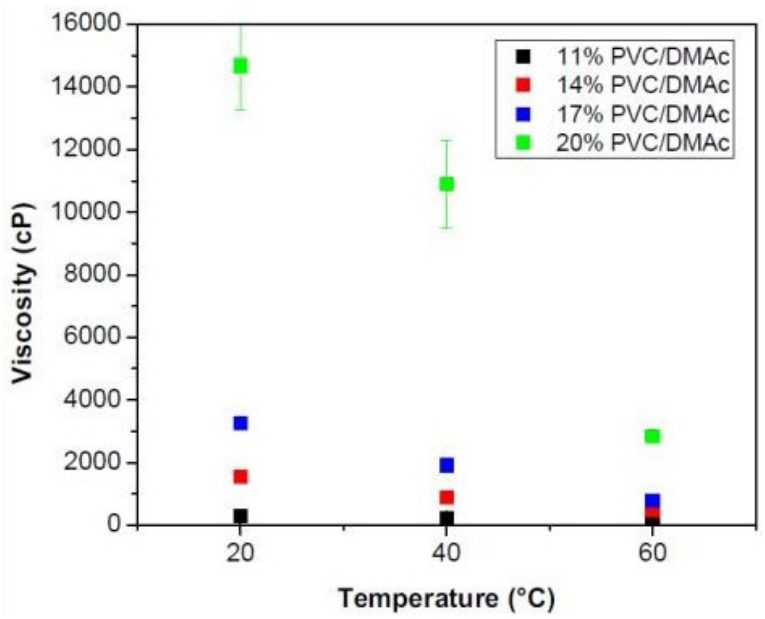

Figure 4. Viscosity of polymeric solutions of PVC in DMAc at different temperatures. 


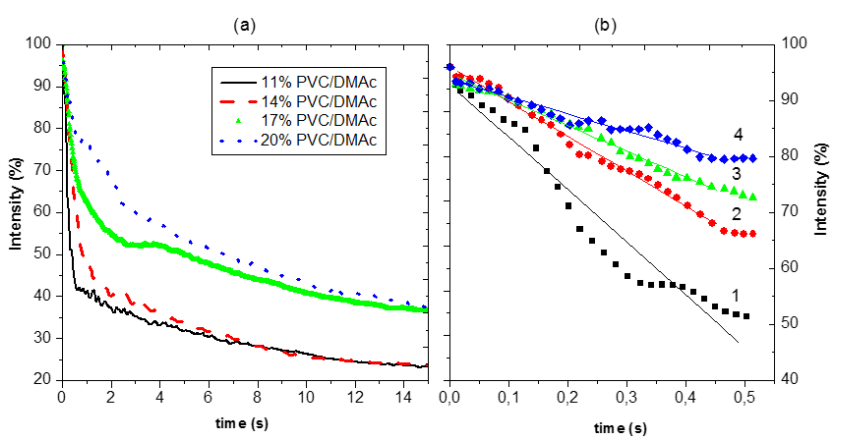

Figure 5. Reduction in the light transmission intensity of PVC/DMAc solutions as a function of immersion time in a water precipitation bath. (a) Light transmittance intensity decay. (b) Linear fit for the first 0.5 seconds.

initial 0.5 seconds after immersion into the water bath. A linear fit of these data indicates a rise in angular coefficient as the polymer concentration increases in the solution, denoting a lower precipitation rate. These data are compiled in Table 2 together with the transport and separation properties. Solutions with lower polymer concentration present smaller angular coefficients, i.e., higher precipitation rate, which favors macrovoid formation and, thereafter, high values of water permeability. Conversely, a decrease in water permeability for the polymer solution with higher PVC concentration indicates reduction in the membrane pore interconnectivity. The BSA rejection for 11 and $14 \mathrm{wt} \%$ PVC membranes was approximately similar, close to $80 \%$. A huge reduction in the permeability was observed for BSA solution indicating high interaction of the protein with the PVC membrane surface.

These results are the first impressions of the membrane morphology behavior associating angular coefficients with precipitation rate. Fen'ko and coworkers (2010) observed the same behavior for PVDFDMAc systems. They showed an increasing in angular coefficient of linear fittings as the PVDF concentration increased in the polymeric solution, estimated for the first 10 seconds after immersion. They also presented similar results adding PEG to the PVDF-DMAc solution.

\section{Membrane formation: effect of additives}

The presence of additives in the polymer solution changes phase separation behavior, as shown in Table 1, as well as may change the precipitation path modifying the membrane morphology. In this study, PVP and $\mathrm{LiNO}_{3}$ were evaluated as additives in a solution with $11 \mathrm{wt} \%$ of PVC, by measuring the light transmittance decay after immersion in the water precipitation bath and observing the final membrane morphology and transport properties.

Figures 6 and 7 show the photomicrographs of membrane cross-sections obtained from PVC solutions with different concentrations of PVP and $\mathrm{LiNO}_{3}$, respectively. The addition of PVP into the PVC solution changes the size and direction of macrovoids, as seen in Figure 6(a) and 6(b).

Adding $10 \mathrm{wt} \%$ of PVP into the solution causes a reduction in macrovoid formation, particularly near the bath interface, favoring sponge-like morphology (Figure 6(c)). These results were expected analyzing the cloud point data in Table 1, which shows reduction in the miscibility region of the system.

Boom et al. (1992) reported that, after immersion in the precipitation bath, a high molar mass polymer additive does not have enough mobility in the initial stages of the precipitation hindering the nucleation

Table 2. Precipitation rate of PVC solution and membrane transport properties.

\begin{tabular}{|c|c|c|c|c|}
\hline $\begin{array}{l}\text { PVC Content } \\
(w t \%)\end{array}$ & $\begin{array}{c}\begin{array}{c}\text { Angular Coefficients } \\
\left({ }^{\mathbf{a}}\right.\end{array} \\
\left(\mathrm{s}^{-1}\right)\end{array}$ & $\begin{array}{c}\mathbf{J}_{\mathrm{w}} \\
\left(\mathrm{L}^{-h^{-1}} \mathbf{m}^{-2} \cdot \mathrm{bar}^{-1}\right)\end{array}$ & $\begin{array}{c}\mathbf{J}_{\mathrm{BSA}} \\
\left(\mathbf{L}^{-h^{-1}} \mathbf{m}^{-2} \cdot \text { bar }^{-1}\right) \\
\end{array}$ & $\begin{array}{l}R \\
(\%)\end{array}$ \\
\hline 11 & $-116 \pm 5$ & $101 \pm 40$ & $12 \pm 1$ & $78 \pm 5$ \\
\hline 14 & $-78 \pm 2$ & $89 \pm 38$ & $13 \pm 1$ & $80 \pm 5$ \\
\hline 17 & $-57 \pm 1$ & $<0.5$ & NR & NR \\
\hline 20 & $-38 \pm 1$ & $<0.5$ & NR & NR \\
\hline
\end{tabular}

a from linear fit of light transmittance intensity decay for 0.5 seconds after immersion in a water bath; NR - Not relevant.

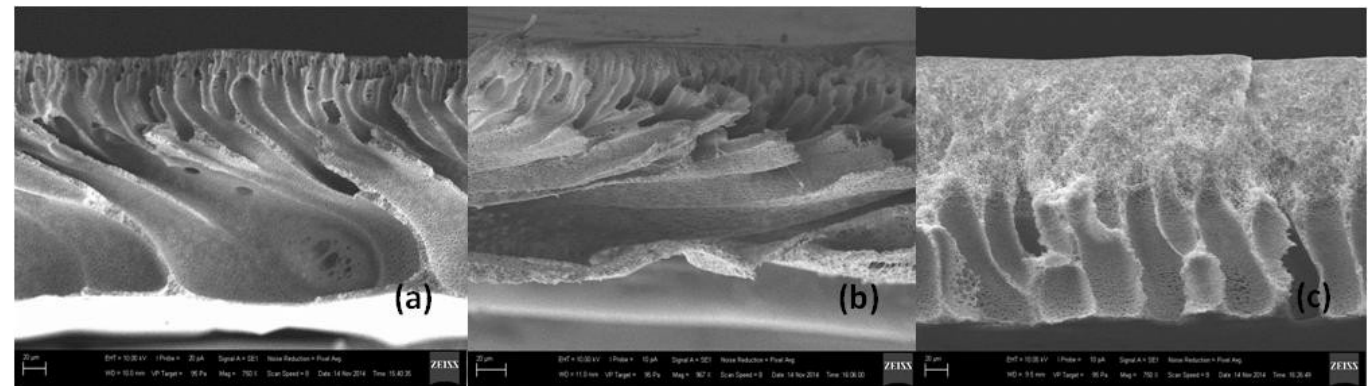

Figure 6. Cross section morphology of the membranes synthesized from $11 \mathrm{wt} \%$ PVC/DMAc system adding (a) $5 \mathrm{wt} \%$, (b) $7 \mathrm{wt} \%$ and (c) $10 \mathrm{wt} \%$ of PVP and instantaneously immersed in a precipitation bath immediately after casting. 


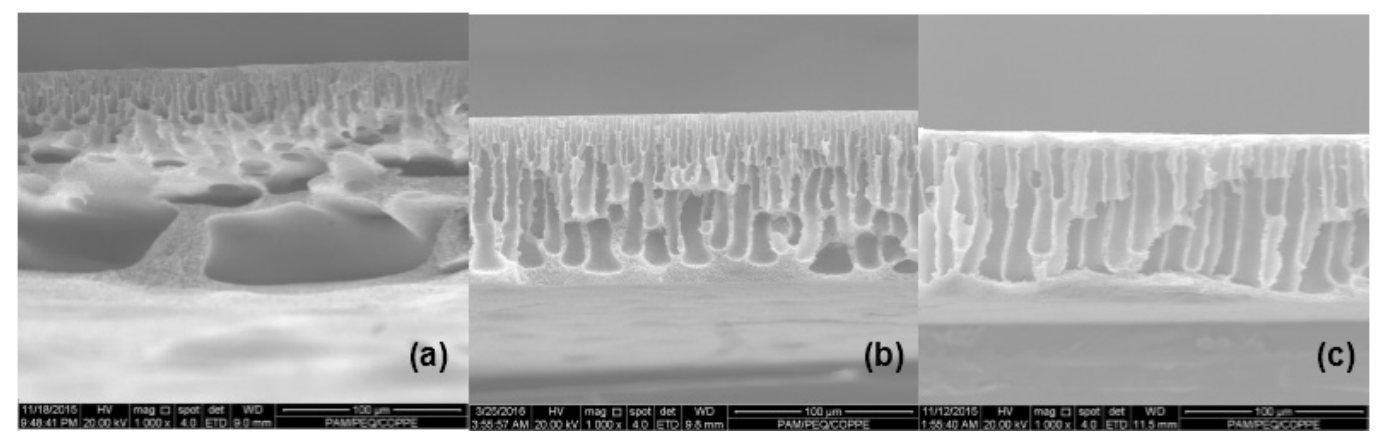

Figure 7. Cross section morphology of the membranes synthesized from $11 \mathrm{wt} \% \mathrm{PVC} / \mathrm{DMAc}$ system adding (a) 3 wt $\%$, (b) $5 \mathrm{wt} \%$ and (c) $7 \mathrm{wt} \%$ of LiNO3 and instantaneously immersed in a precipitation bath immediately after casting.

mechanism. In other words, high solvent and nonsolvent affinities lead the system to precipitate directly into the spinodal region in the phase diagram, avoiding the metastable region in which the nucleation takes place. High pore interconnectivity is expected from phase separation by spinodal demixing. When lithium nitrate was used as additive in the PVC solution a different effect was observed in the membrane morphology. At low $\mathrm{LiNO}_{3}$ concentration the enlargement of macrovoids is shown in Figure 7(a), but a slight increase in its concentration ( 3 to $5 \mathrm{wt} \%$ ) leads to more organized and vertical oriented macrovoids (Figures 7(b) and 7(c)). It might be the consequence of higher polymer concentration at the precipitation onset and reduction in miscibility of the system.

The presence of additive in the polymer solution also changes the viscosity, as observed in Figure 8. As previously discussed, high solution viscosity values slow down the initial mass transfer exchange between the polymer solution and the precipitation bath, leading to lower polymer concentration at the precipitation onset. It is expected that this condition reduces the mass transfer resistance between the precipitation bath and underneath layer in the PVC solution, favoring spongelike morphologies and reduction in macrovoid formation.

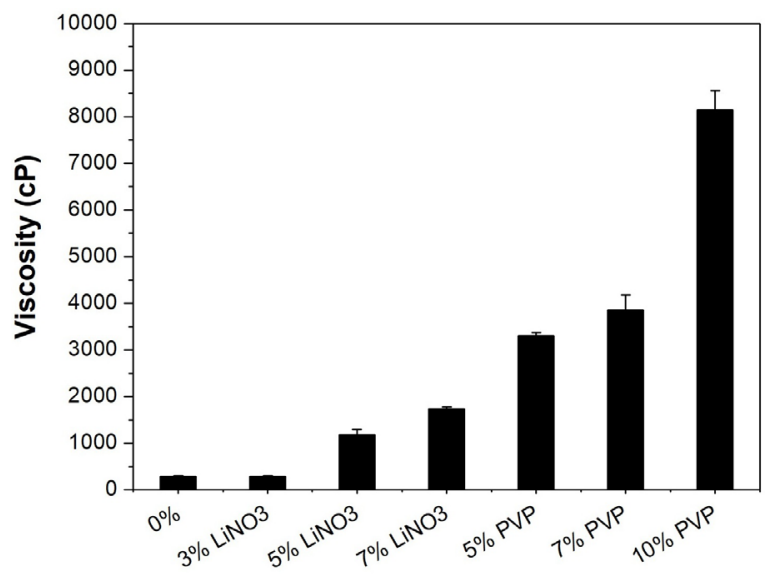

Additive Content (\%)

Figure 8. Effect of additive concentration on the viscosity of $11 \mathrm{wt} \% \mathrm{PVC}$ solution.
The light transmission decay of PVC solutions with different additives is presented in Figure 9 and the initial precipitation rates are shown in Table 3. This table also presents the membrane transport properties.

As seen in Figure 9, as the additive concentration in the solution increases, the precipitation rate decreases, indicating a delay in mass exchange between solvent and non-solvent. However, a different solution precipitation behavior is clearly observed for each additive. PVP reduces the light transmittance, but the precipitation of the solution starts immediately after immersion into the water bath, while a delay time is observed for solutions with $\mathrm{LiNO}_{3}$. It could mean that the main effect of PVP is related to higher viscosity and low chain mobility, reducing mass transfer rate and favoring spinodal demixing.

On the other hand, for $\mathrm{LiNO}_{3}$ a delay in precipitation would allow an increase in polymer concentration at the solution top layer before the precipitation onset, which creates an extra resistance for mass transfer between the water bath and the solution below, favoring macrovoid formation. The delay promoted by $\mathrm{LiNO}_{3}$ as additive could be related with some possible gelation of the top layer due to a formation of a complex between the salt and DMAc. However, more experiments should be performed to try to separate in ternary diagrams the composition that leads the solution to the gel phase or liquid phase.

The initial precipitation rates for both additives and for the solution without additive are shown in Table 3,

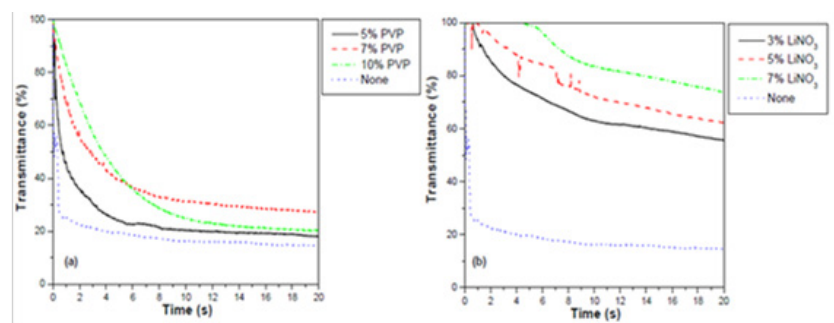

Figure 9. Reduction in the light transmission intensity of $11 \mathrm{wt} \% \mathrm{PVC}$ solution with different additive content as a function of the immersion time in a water precipitation bath. (a) PVP; (b) $\mathrm{LiNO}_{3}$. 
Table 3. Effect of different PVP and $\mathrm{LiNO}_{3}$ concentrations on precipitation rate of $11 \mathrm{wt} \% \mathrm{PVC}$ solution and membrane transport properties.

\begin{tabular}{|c|c|c|c|c|c|c|}
\hline \multicolumn{2}{|c|}{$\begin{array}{c}\text { Additive } \\
\text { content (\%wt) }\end{array}$} & $\begin{array}{c}\text { Exposure } \\
\text { Time }\end{array}$ & $\begin{array}{c}\text { Angular } \\
\text { coefficient }\end{array}$ & $\begin{array}{c}J_{\mathrm{w}} \\
\left(\mathbf{L}^{-h^{-1}} \mathrm{~m}^{-2} \cdot \text { bar }^{-1}\right)\end{array}$ & $\begin{array}{c}\mathbf{J}_{\mathrm{BSA}} \\
\left(\mathbf{L} \cdot \mathrm{h}^{-1} \mathbf{m}^{-2} \cdot \mathrm{bar}^{-1}\right)\end{array}$ & $\begin{array}{c}\text { BSA } \\
\text { Rejection (\%) }\end{array}$ \\
\hline \multirow{4}{*}{\multicolumn{2}{|c|}{ None }} & Instantaneous & $-116 \pm 5$ & $101 \pm 40$ & $12 \pm 1$ & $78 \pm 5$ \\
\hline & & $30 \mathrm{~s}$ & $-123 \pm 2$ & $87 \pm 11$ & $64 \pm 5$ & $80 \pm 5$ \\
\hline & & $60 \mathrm{~s}$ & $-127 \pm 3$ & $82 \pm 20$ & $22 \pm 5$ & $82 \pm 5$ \\
\hline & & Infinite & $-0.8 \pm 0.2$ & $2 \pm 1$ & $<0.5$ & NR \\
\hline \multirow{12}{*}{ PVP } & \multirow{4}{*}{5} & Instantaneous & $-98 \pm 25$ & $257 \pm 58$ & $14 \pm 5$ & $90 \pm 5$ \\
\hline & & $30 \mathrm{~s}$ & $-53 \pm 4$ & $263 \pm 73$ & $18 \pm 5$ & $71 \pm 5$ \\
\hline & & $60 \mathrm{~s}$ & $-64 \pm 8$ & $286 \pm 109$ & $22 \pm 5$ & $76 \pm 5$ \\
\hline & & Infinite & $-2 \pm 1$ & $<2$ & NR & NR \\
\hline & \multirow{4}{*}{7} & Instantaneous & $-33 \pm 8$ & $171 \pm 24$ & $18 \pm 5$ & $80 \pm 5$ \\
\hline & & $30 \mathrm{~s}$ & $-23 \pm 2$ & $160 \pm 27$ & $53 \pm 5$ & $66 \pm 5$ \\
\hline & & $60 \mathrm{~s}$ & $-21 \pm 3$ & $162 \pm 29$ & $38 \pm 5$ & $44 \pm 5$ \\
\hline & & Infinite & $-4 \pm 1$ & $<2$ & NR & NR \\
\hline & \multirow{4}{*}{10} & Instantaneous & $-17 \pm 3$ & $150 \pm 14$ & $67 \pm 5$ & $68 \pm 5$ \\
\hline & & $30 \mathrm{~s}$ & $-15 \pm 3$ & $165 \pm 39$ & $42 \pm 5$ & $50 \pm 5$ \\
\hline & & $60 \mathrm{~s}$ & $-14 \pm 4$ & $148 \pm 22$ & $34 \pm 5$ & $82 \pm 5$ \\
\hline & & Infinite & $-0.3 \pm 0.2$ & $<2$ & NR & NR \\
\hline \multirow{12}{*}{$\mathrm{LiNO}_{3}$} & \multirow{4}{*}{3} & Instantaneous & $-24 \pm 8$ & $306 \pm 51$ & $20 \pm 5$ & $65 \pm 5$ \\
\hline & & $30 \mathrm{~s}$ & $-9 \pm 2$ & $328 \pm 65$ & $13 \pm 5$ & $90 \pm 5$ \\
\hline & & $60 \mathrm{~s}$ & $-12 \pm 6$ & $361 \pm 33$ & $14 \pm 5$ & $85 \pm 5$ \\
\hline & & Infinite & $-0.5 \pm 0.2$ & $<8$ & NR & NR \\
\hline & \multirow{4}{*}{5} & Instantaneous & $-15 \pm 5$ & $310 \pm 77$ & $17 \pm 5$ & $53 \pm 5$ \\
\hline & & $30 \mathrm{~s}$ & $-32 \pm 15$ & $170 \pm 76$ & $16 \pm 5$ & $22 \pm 5$ \\
\hline & & $60 \mathrm{~s}$ & $-51 \pm 4$ & $286 \pm 84$ & $2 \pm 0.5$ & $24 \pm 5$ \\
\hline & & Infinite & $-0.2 \pm 0.1$ & $40 \pm 5$ & NR & NR \\
\hline & \multirow{4}{*}{7} & Instantaneous & $-6 \pm 1$ & $1,348 \pm 62$ & $18 \pm 5$ & $69 \pm 5$ \\
\hline & & $30 \mathrm{~s}$ & $-5 \pm 1$ & $389 \pm 168$ & $10 \pm 5$ & $49 \pm 5$ \\
\hline & & $60 \mathrm{~s}$ & $-3 \pm 1$ & $545 \pm 68$ & $18 \pm 5$ & $34 \pm 5$ \\
\hline & & Infinite & $-0.9 \pm 0.2$ & $110 \pm 5$ & NR & NR \\
\hline
\end{tabular}

${ }^{a}$ From linear fit of light transmittance intensity decay for 0.5 seconds after immersion in a water bath; NR - Not relevant.

which shows an accentuated reduction of this parameter when the additive concentration increases. This effect was more severe for $\mathrm{LiNO}_{3}$, probably due to the increase in the polymer concentration at the solution top layer during the delay time for the precipitation onset. An augment was observed in the permeability of water and BSA solution when additives were used in the polymer solution. However, the difference in precipitation mechanism of each additive leads to a distinct behavior with concentration in the polymer solution. Whereas PVP promotes an initial increase in water permeability followed by a continuous reduction of it, the presence of higher amounts of $\mathrm{LiNO}_{3}$ leads to a continuous increase in water permeability. Hence, it is also possible to conclude that these results corroborate the previous discussion about membrane morphology.

Some authors report similar results for different systems, attributing changes in membrane morphology to the reduction in system miscibility, solution viscosity and precipitation rate (Mei et al., 2011; Yoo et al., 2004). Yoo et. al. (2004) also point out that PVP addition to the polymer solution may present opposite effects on membrane morphology as a function of its molar mass and interaction with solvent and polymer.

WithPVPasadditive, BSArejectionexhibited similar behavior to that observed with water permeability.
However, BSA rejection was approximately constant when $\mathrm{LiNO}_{3}$ was used. It is important to stress that BSA rejection was higher for membranes prepared from solutions with low PVP concentration than from solutions without additive or with $\mathrm{LiNO}_{3}$. Another interesting effect is an increase in BSA solution permeability with augment in PVP concentration in the polymer solution, which is probably related with higher hydrophilicity and reduction in BSA adsorption due to the presence of PVP in the membrane matrix due to a polymer entanglement effect (Borges, 1993).

\section{Membrane formation: Ambient exposure time before immersion into the water bath}

The exposure time to the environment before immersion into the water bath plays a significant role in membrane morphology, because it can allow water vapor (air humidity) penetration into the polymeric film almost without solvent outflow, creating favorable conditions for pore formation in the top layer. The solution was instantaneously immersed into the precipitation bath or exposed to the ambient for $30 \mathrm{~s}, 60 \mathrm{~s}$ or until complete precipitation by moisture absorption (infinite time). All membrane syntheses were monitored by light transmittance decay as the precipitation proceeded. 
Figure 10 shows the cross-section morphology of membranes prepared from $11 \mathrm{wt} \%$ PVC solution with different exposure times before immersion in the water bath. As can be seen, the increase of exposure time to the ambient results in enlargement of the macrovoids and some loss in orientation, favoring sponge-like morphologies at infinite exposure time. Table 3 shows a slight reduction in water permeability, which may be the consequence of higher membrane compaction during permeation due to large macrovoids in its cross-section.
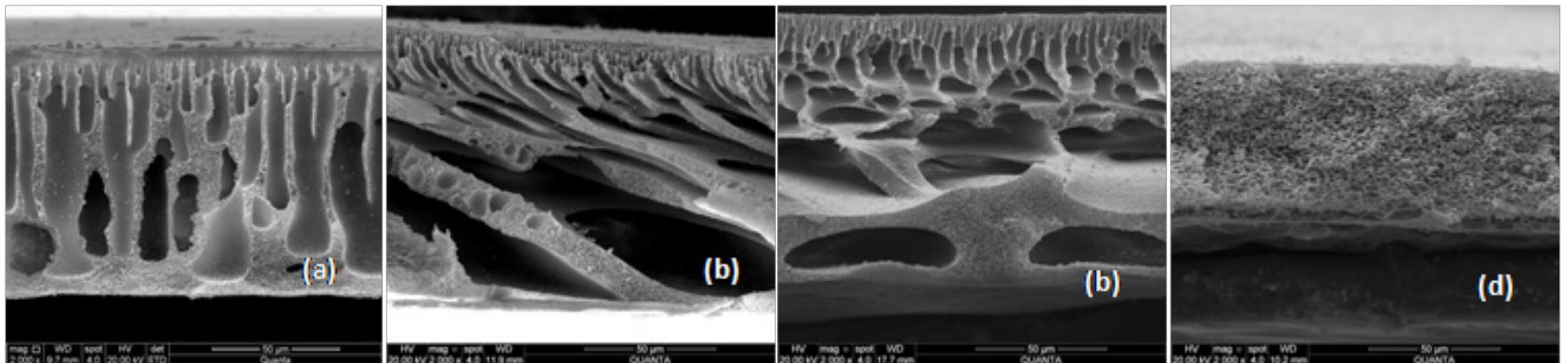

Figure 10. Cross section morphology of membranes synthesized from $11 \mathrm{wt} \%$ PVC/DMAc system with exposure time before immersion: (a) instantaneous, (b) 30s, (c) 60s and (d) infinite.
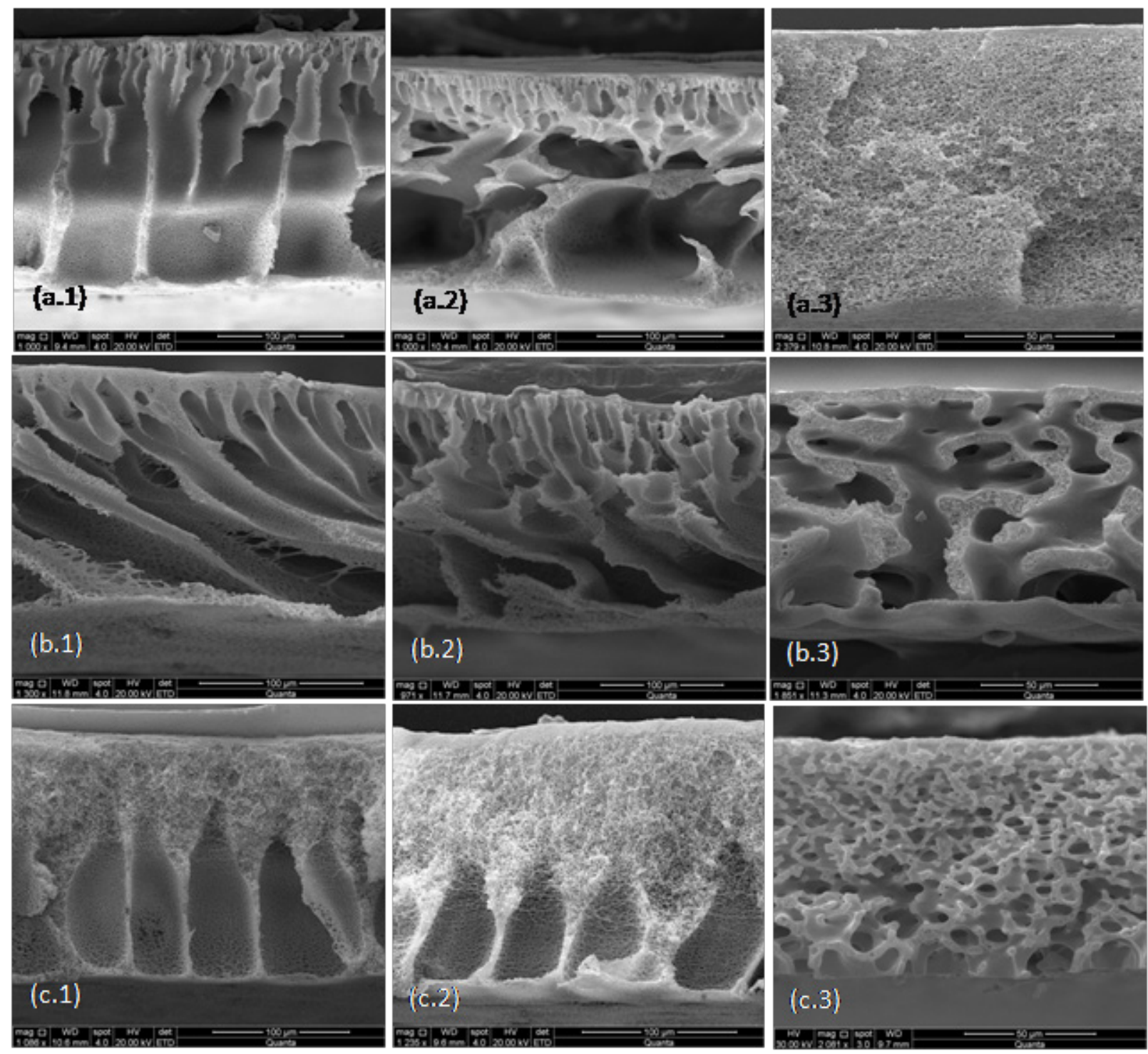

Figure 11. Cross section morphology of the membranes synthesized from $11 \mathrm{wt} \%$ PVC/DMAc system adding (a) 5 $\mathrm{wt} \%$, (b) $7 \mathrm{wt} \%$ and (c) $10 \mathrm{wt} \%$ of PVP immersed in a water bath with exposure time before immersion of (1) $30 \mathrm{~s}$, (2) $60 \mathrm{~s}$ and (3) infinite. 
However, it also shows a higher permeability for BSA solution, keeping the value of rejection, which could be a result of large pores in the membrane top layer and BSA adsorption phenomenon. Furthermore, it was observed that the precipitation rate was almost constant with exposure time, indicating that water absorption by the PVC solution did not change the precipitation path after immersion into the bath. Thus, changes in membrane morphology and transport properties may be mainly attributed to water absorption by the PVC solution during the exposure time, creating conditions to favor nucleation of a lean polymer phase and larger pores at the membrane surface. This effect also creates an extra resistance to mass transfer between the PVC solution and the water bath, providing conditions for nuclei expansion and enlargement of macrovoids.
Figures 11 and 12 show the influence of PVP and $\mathrm{LiNO}_{3}$, respectively, as additive to the PVC solution on membrane cross-section morphology as a function of the exposure time before immersion into the water bath. Higher water absorption by the solution is expected as the exposure time increases, which could accelerate the precipitation at the solution top layer, creating an additional mass transfer resistance. This condition favors the coalescence of nascent macrovoids and formation of large cavities in the membrane sub layer. At $10 \mathrm{wt} \%$ of PVP this is not observed, probably due to higher solution viscosity and slower water penetration by diffusion into deeper regions of the solution. For solutions with high PVP content, a prolonged exposure to the environment allows slow precipitation of the solution with low water content, which changes the

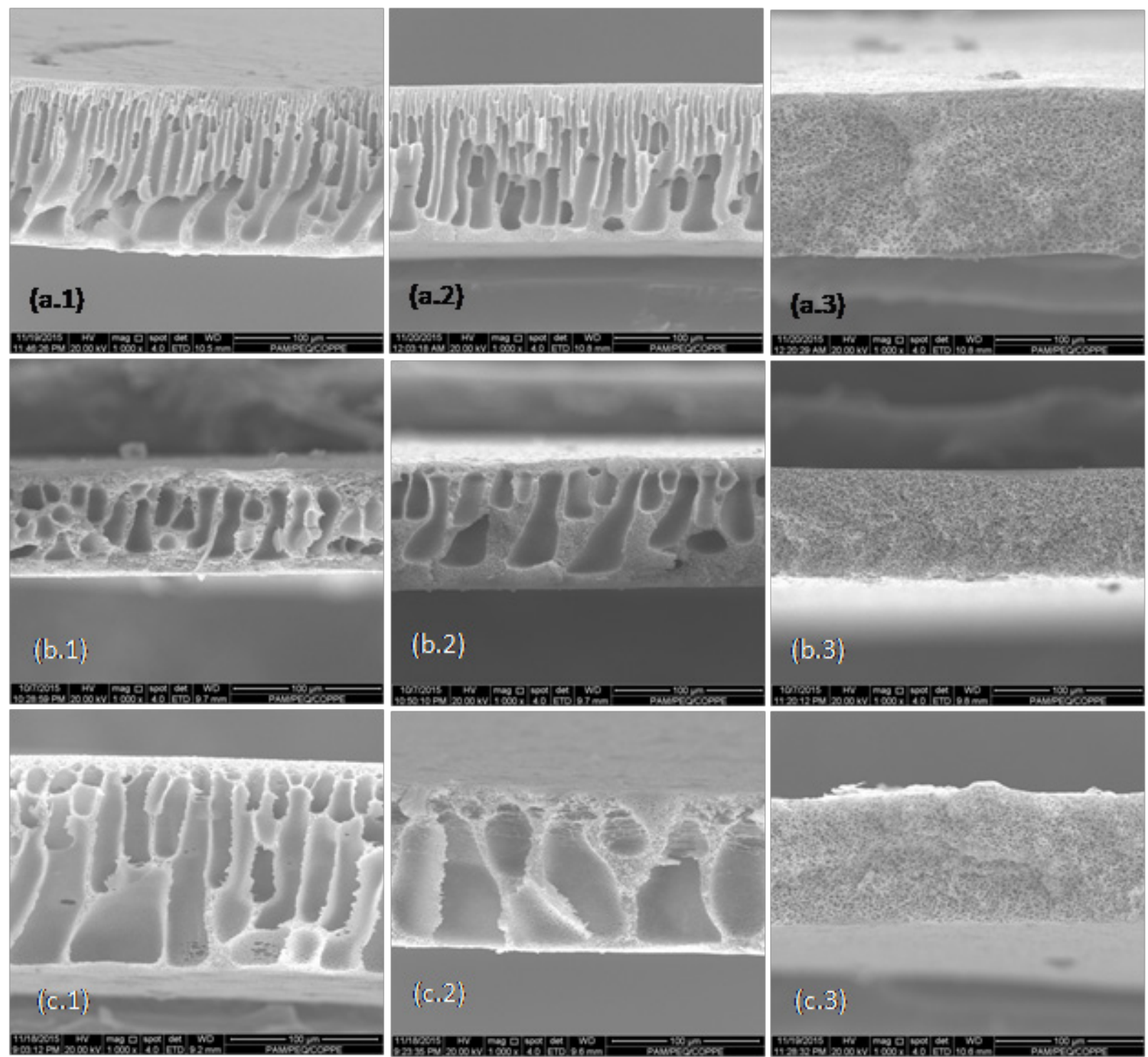

Figure 12. Cross section morphology of the membranes synthesized from $11 \mathrm{wt} \%$ PVC/DMAc system adding (a) 3 $\mathrm{wt} \%$, (b) $5 \mathrm{wt} \%$ and (c) $7 \mathrm{wt} \%$ of $\mathrm{LiNO}_{3}$ immersed in a water bath with exposure time before immersion of (1) $30 \mathrm{~s}$, (2) $60 \mathrm{~s}$ and (3) infinite. 
phase separation mechanism forming interconnected phases, which could characterize spinodal demixing.

For PVP as additive, Table 3 shows reduction in the initial precipitation rate as exposure time increases, indicating slower exchange between solvent and nonsolvent; which corroborates our previous discussion about membrane morphology. The effect of PVP at different exposure times on transport properties was similar to that observed for instantaneous immersion, evidencing the competition between membrane hydrophilicity and pore size distribution. Except for infinite time exposure, a large variation of the water permeability with higher exposure interval was not observed, which could be the result of top layer precipitation before immersion in the water bath. Thus, reduction in BSA rejection could also be related to growth of previously formed nuclei during the exposure time.

For membranes prepared from PVC solution adding $\mathrm{LiNO}_{3}$ as additive, the increase of exposure time before immersion into the water bath favors macrovoid growth. However, when the exposure was prolonged (infinite) sponge-like pores were formed, as seen in Figure 12. This behavior becomes more evident as the additive concentration increases in PVC solution.

Table 3 shows that the initial precipitation rates for solutions with $\mathrm{LiNO}_{3}$ as additive, in general, were lower than those observed for solutions without additive or with PVP. It is an indication of an additional top layer resistance promoted by water vapor absorption, similar to that observed for PVP, but with higher intensity. The presence of $\mathrm{LiNO}_{3}$ in the PVC solution and instantaneous immersion in the precipitation bath leads to the highest values of water permeability, which is probably related to the proximity of the phase separation region and high initial nucleation rate at the solution/bath interface. The behavior of permeability and $\mathrm{BSA}$ rejection with exposure time is again related to large pores at the membrane surface due to water vapor absorption and membrane compaction during permeation due to large macrovoids in the membrane cross-section.

\section{CONCLUSIONS}

As a general conclusion, when the PVC solution is closer to the phase separation region nuclei growth is not favored and the membrane will present spongelike morphology. However, precipitation of a solution layer near the bath interface delays solvent and nonsolvent exchange, favoring macrovoid formation in the layers beneath the solution/bath interface. This condition was confirmed by measuring the initial precipitation rate by light transmittance decay. The same effect was observed in the presence of PVP or
$\mathrm{LiNO}_{3}$ as additives in the PVC solution. However, PVP favors instantaneous precipitation, while with $\mathrm{LiNO}_{3}$ precipitation delay was observed. In both situations, water permeability increases with additives in the PVC solution, which indicates formation of a porous top layer in both mechanisms. On the other hand, whereas PVP promotes an initial increase in water permeability followed by a continuous reduction in it, the presence of higher amounts of $\mathrm{LiNO}_{3}$ leads to a continuous increase in water permeability. Hence, it is also possible to conclude that instantaneous demixing promoted by PVP addition will suppress macrovoids with increasing PVP concentration in the PVC solution. In contrast, the precipitation delay observed with $\mathrm{LiNO}_{3}$ still predominates at higher additive concentration, maintaining macrovoids in the membrane sub-layer and leading to higher water permeabilities. A sponge-like morphology only was observed with $\mathrm{LiNO}_{3}$ as additive when a prolonged exposure time to ambient was allowed, which was related to slow water vapor absorption by the solution. Conversely, when PVP was used as additive this condition leads to interconnected pores, suggesting a spinodal demixing mechanism.

The BSA rejection behavior corroborates these conclusions, i.e., it was always high with PVP content and diminished when increasing $\mathrm{LiNO}_{3}$ in the PVC solution. The transport properties obtained for membranes prepared with $\mathrm{LiNO}_{3}$ are the consequence of larger surface pores generated by the water vapor absorption and due to macrovoids formed in its crosssection.

Membranes produced with smaller PVC content and additive showed higher permeability values, but the solute rejection behavior seems to depend of other factors, such as the presence of superficial pores, macrovoid formation and membrane hydrophilicity. The light transmittance experiments can give an idea of the precipitation rate and seem to be correlated with water permeability values. However, more results need to be obtained targeting membrane transport properties optimization.

Thus, the NIPS technique is suitable to produce PVC membranes with different morphologies by changes in either the thermodynamic properties as well as the kinetic parameters. It produced a closed membrane with very slow water permeability from solutions with high PVC content and no additive present or an open one with the highest water permeability value $\left(\sim 1,350 \mathrm{~L} \cdot \mathrm{h}^{-1} \cdot \mathrm{m}^{-2} \cdot \mathrm{bar}^{-1}\right)$ by using $5 \mathrm{wt} \%$ of $\mathrm{LiNO}_{3}$ and instantaneous immersion in the precipitation bath.

\section{SYMBOLS AND UNITS}

$\begin{array}{ll}\text { BSA } & \text { Bovine serum albumin } \\ \text { DMAc } & \text { N,N-dimethylacetamide }\end{array}$




$\begin{array}{ll}\text { DMF } & \text { N,N-dimethylformamide (DMF) } \\ \mathrm{J}_{\mathrm{w}} & \text { Water permeability, } \mathrm{L}^{-1} \cdot \mathrm{m}^{-2} \cdot \mathrm{bar}-1 \\ \mathrm{~J}_{\text {BSA }} & \text { BSA solution permeability, } \mathrm{L}^{-1} \cdot \mathrm{m}^{-2} \cdot \mathrm{bar}^{-1} \\ \mathrm{LiNO}_{3} & \text { Lithium nitrate } \\ \mathrm{NIPS} & \text { Non-solvent induced phase separation } \\ & \text { process } \\ \mathrm{NMP} & \text { N-methyl-pirrolydone } \\ \mathrm{PES} & \text { Polyethersulfone } \\ \mathrm{PEI} & \text { Polyetherimide } \\ \mathrm{PEG} & \text { Polyethylene glycol } \\ \mathrm{PVB} & \text { Polyvinylbutyral } \\ \mathrm{PVC} & \text { Polyvinylchloride (PVC) } \\ \mathrm{PVP} & \text { Polyvinylpyrrolidone } \\ \mathrm{THF} & \text { Tetrahydrofuran } \\ \mathrm{wt} \% & \text { Weight percent } \\ \mathrm{Q}_{\mathrm{w}} & \text { Volumetric permeate flow rate, } \mathrm{L} / \mathrm{h} \\ \Delta \mathrm{P} & \text { Pressure difference through } \\ & \text { the membrane, bar } \\ \mathrm{A}_{\mathrm{A}} & \text { Membrane surface area, } \mathrm{m}^{2} \\ \mathrm{Q}_{\mathrm{w}} / \mathrm{A} & \text { Water permeation flux, } \mathrm{L} \mathrm{m}^{-2} \mathrm{~h}^{-1} \\ \mathrm{R}^{-1} & \text { BSA rejection, \% } \\ \mathrm{C}_{\mathrm{p}} & \text { BSA concentration in permeate, } \mathrm{g} / \mathrm{mL} \\ \mathrm{C}_{\mathrm{f}} & \text { BSA concentration in feed solution, } \mathrm{g} / \mathrm{mL}\end{array}$

\section{REFERENCES}

Babu, P. R., Gaikar, V. G., Preparation, Structure, and Transport Properties of Ultrafiltration Membranes of Poly(vinyl chloride) (PVC), Carboxylated Poly(vinyl chloride) (CPVC), and PVC/CPVC Blends, Journal of Applied Polymer Science, 73, 1117 - 1130 (1999). https://doi.org/10.1002/ (SICI)1097-4628(19990815)73:7\%3C1117::AIDAPP3\%3E3.0.CO;2-H

Babu, P. R., Gaikar, V. G., Preparation, Structure, and Transport Properties of Ultrafiltration Membranes of Poly(vinyl chloride) and Poly(vinyl pyrrolidone) Blends. Journal of Applied Polymer Science, 77, 2606 - 2620 (2000). https://doi.org/10.1002/1097$4628(20000919) 77: 12 \% 3$ C2606::A ID APP80\%3E3.0.CO;2-Z

Baker, R. W.; Membrane Technology and Aplications. $2^{\text {nd }}$ ed.; John Wiley \& Sons Ltda (2004).

Barzin, J., Sadatnia, B., B. Correlation between macrovoid formation and the ternary phase diagram for polyethersulfone membranes prepared from two nearly similar solvents. Journal of Membrane Science, 325, 92 - 97 (2008). https:// doi.org/10.1016/j.memsci.2008.07.003

Boom, R. Membrane formation by immersionprecipitation: The role of polymeric additive. University of Twente, Twente, (1992).

Bodzek, M., Konieczny, K., The influence of molecular mass of poly (vinyl chloride) on the structure and transport characteristics of ultrafiltration membranes. Journal of Membrane Science, 61, 131 - 156 (1991). https://doi.org/10.1016/03767388(91)80011-T

Borges, C. P. Fibras Ocas Compostas para Remoção de Poluentes Orgânicos Voláteis de Soluções Aquosas. $\mathrm{Ph}$. D. Thesis, Federal University of Rio de Janeiro, Rio de Janeiro (1993).

Carvalho, R. B. Fibras Ocas Compostas para Nanofiltração e Osmose Inversa preparadas pela técnica de Precipitação por Imersão de duas Soluções Poliméricas Extrusadas simultaneamente. $\mathrm{Ph}$. D. Thesis, Federal University of Rio de Janeiro, Rio de Janeiro, RJ (2005).

Fen'ko, L. A., Semenkevich, N. G., Bil'dy, A. V., The Kinetics of Membrane Pore Structure Formation by Phase Inversion. Petroleum Chemistry, 51, 527-535 (2011). https://doi.org/10.1134/ S0965544111070073

Frommer, A., Messalem, R. M., Mechanism of Membrane Formation.VI.Convective Flows and Large Void Formation during Membrane Precipitation. Ind. Eng. Chem. Prod. Res. Develop., 12, 328-333 (1973). https://doi.org/10.1021/ i360048a015

Guillen, G. R., Pan Y., Li M. and Hoek M. V., Preparation and characterization of membrane formed by Nonsolvent Induced Phase Separation: A Review. Industrial \& Engineering Chemistry Research, 50, 3798-3817 (2011). https://doi. org/10.1021/ie101928r

Gullotin, M., Lemoyne, C., Noel, C. and Monnerle, L., Physicochemical processes occurring during the formation of cellulose diacetate membranes, research of criteria for optimizing membrane performance. IV. Cellulose diacetate-acetoneorganic additive casting solutions. Desalination, 21, 165-181 (1977). https://doi.org/10.1016/S00119164(00)80314-8

Hirose, S., Hayashi, M. Tamura, N., Preparation and Structures of the Poly(vinyl chloride) Porous Membranes. Journal of Molecular Catalysis, 6, 251 - 260 (1979). https://doi.org/10.1016/03045102(79)80031-0

Hirose, S., Yasukawa, E. and Hayashi, M., Wet Poly(vinyl chloride) Membrane as a support: sorption and transport of low-molcecular-weight organic compouds and proteins. Journal of Membrane Science, 11, 177 - 185 (1982). https:// doi.org/10.1016/S0376-7388(00)81400-3

Kaway, T., Lee, Y. M., Preparation of asymmetric porous membranes of poly(vinyl chloride). Polymer, 38, 1631 - 1637 (1997). https://doi. org/10.1016/S0032-3861(96)00662-3

Kools, W. F. C. Membrane Formation by Phase Inversion in Multicomponent Polymer Systems, Mechanisms and Morphologies. PhD Thesis, University of Twente, Twente, 1998. 
Machado, P. S. T., Habert, A. C., Borges, C. P., Membrane formation mechanism based on precipitation kinetics and membrane morphology: flat and hollow fiber polysulfone membranes. Journal of Membrane Science, 155, 171 183 (1999). https://doi.org/10.1016/S03767388(98)00266-X

Maghsoud, Z., Famili, M. H. N., Madaeni, S. S., Preparation of Polyvinylchloride Membranes from Solvent Mixture by Immersion Precipitation. Journal of Applied Polymer Science, DOI: 10.1002/ app.40206 (2014). https://doi.org/10.1002/ app.40206

Mckelvey, A., Koros, W. J., W. J. Phase separation, vitrification, and the manifestation of macrovoids in polymeric asymmetric membranes. Journal of Membrane Science, 112, 29-39 (1996). https://doi. org/10.1016/0376-7388(95)00197-2

Mei, S., Xiao, C., Hu, X., Preparation of Porous PVC Membrane via a Phase Inversion Method from PVC/DMAc/Water/Additives. Journal of Aplied Polymer Science, 120, 557-562 (2011). https://doi. org/10.1002/app.33219

Mulder, M.; Basic Principles of Membrane Technology. Kluwer Academic Publishers (1991). https://doi. org/10.1007/978-94-017-0835-7

Okuno, H., Renzoa, K., Uragamib, T., Influence of casting solution additive, degree of polymerization, and polymer concentration on poly (vinyl chloride) membrane properties and performance. Journal of Membrane Science, 83, 199 - 209 (1993). https:// doi.org/10.1016/0376-7388(93)85267-Z

Peng, Y., Sui, Y., Compatibility research on PVC/PVB blended membranes. Desalination, 196, 13 - 21 (2006). https://doi.org/10.1016/j.desal.2005.07.053

Porter, M. C.; Handbook of Industrial Membrane Technology (1990)

Reuvers, B. Membrane formation. Diffusion induced demixing process in ternary polymeric systems. $\mathrm{Ph}$. D. Thesis, University of Twente, Twente (1987).
Smolders, C. A., Reuvers, A. J., Boom, R. M. and Wienk I. M., Microstructures in phase-inversion membranes.Part 1. Formation of macrovoids. Journal of Membrane Science. Journal of Membrane Science, 73, 259-275 (1992). https:// doi.org/10.1016/0376-7388(92)80134-6

Strathmann, H., Kock, K., Amar, P., The formation mechanism of asymmetric membranes. Desalination, 16, 179 - 203 (1975). https://doi. org/10.1016/S0011-9164(00)82092-5

Uragami, T., Ohsumi, Y., Sugi, M., Permeabilities of special polymer membranes: 38. Formation mechanism of finger-like cavities in membranes from cellulose nitrate and single solvent. Polymer, 23, 999-1004 (1982). https://doi.org/10.1016/00323861(82)90400-1

$\mathrm{Xu}, \mathrm{J} ., \mathrm{Xu}, \mathrm{Z} . \mathrm{-L}$., Hydrophilic modification of polyvinylchloride hollow fiber membranes by silica with a weak in situ sol-gel method. Journal of Membrane Science, 208, 203-212 (2002). https:// doi.org/10.1016/S0376-7388(02)00261-2

Wijmans, J. G., Kant J., Mulder M.H. V. and Smolders, C. A., Phase separation phenomena in solutions of polysulfone in mixtures of a solvent and a nonsolvent: relationship with membrane formation. Polymer, 26, 1539-1545 (1985). https:// doi.org/10.1016/0032-3861(85)90090-4

Yoo, H. S., Kim, H. J., Jho, Y. J., Won, J., Kang, S. Y., Influence of the addition of PVP on the morphology of asymmetric polyimide phase inversion membranes: effects of PVP molecular weight. Journal of Membrane Science, 236, 203-207 (2004). https://doi.org/10.1016/j.memsci.2004.02.017

Young, T.-H., Cheng, L.-P., Lin, D.-J., Fane, L. and Chuang W.-Y., Mechanisms of PVDF membrane formation by immersion-precipitation in soft (1-octanol) and harsh (water) nonsolvents. Polymer, 40, 5315-5323 (1999). https://doi.org/10.1016/ S0032-3861(98)00747-2 
